\title{
Erratum to: Flupirtine-induced liver injury-Seven cases from the Berlin Case-control Surveillance Study and review of the German spontaneous adverse drug reaction reporting database
}

\author{
Antonios Douros • Elisabeth Bronder • Frank Andersohn • \\ Andreas Klimpel • Michael Thomae • Hans-Dieter Orzechowski • \\ Reinhold Kreutz • Edeltraut Garbe
}

Published online: 22 January 2015

(C) Springer-Verlag Berlin Heidelberg 2015

Erratum to: Eur J Clin Pharmacol (2014) 70:453-459 DOI 10.1007/s00228-013-1631-4

We would like to bring to your attention a minor error in the article. The age of one of the patients was wrongly stated as " 31 " years old instead of the correct " 54 " years old, making following changes in the article necessary:

- Abstract, Results (page 1): correct is " 58 " and not " 55 "
- Results, Case series (page 2): correct is " $58 \pm 12$ " and not "55 \pm 16 "

- Table 1, column "Patient 3", row "Age" (page 3): correct is "54" and not " 31 "

- Table 4, column "FAKOS", row "Age / mean" (page 5): correct is " 58 " and not " 55 "

- Table 4, column "FAKOS", row "Age / standard deviation" (page 5): correct is " \pm 12 " and not " \pm 16 "

The online version of the original article can be found at http://dx.doi.org/ 10.1007/s00228-013-1631-4.

A. Douros · E. Bronder · A. Klimpel · H.-D. Orzechowski ·

R. Kreutz $\cdot$ E. Garbe

Department of Clinical Pharmacology and Toxicology,

Charité-Universitätsmedizin Berlin, Berlin, Germany

F. Andersohn

Institute for Social Medicine, Epidemiology and Health Economy,

Charité-Universitätsmedizin Berlin, Berlin, Germany

M. Thomae

Department of Surgery, Maria-Heimsuchung Caritas Klinik Pankow,

Berlin, Germany

E. Garbe $(\bowtie)$

Leibniz Institute for Prevention Research and Epidemiology - BIPS,

Achterstr. 30, 28359 Bremen, Germany

e-mail: garbe@bips.uni-bremen.de 